УДК 629.3

DOI: $10.15827 / 0236-235 X .125 .130-133$
Дата подачи статьи: 26.09.18

2019. T. 32. № 1. C. 130-133

\title{
Разработка программно-аппаратного комплекса для удаленной диагностики наземных транспортных средстө nо каналу GSM
}

\author{
Д.С. Аавыгин ${ }^{1}$, к.т.н., старший научный сотрудник \\ B.B. Аевианов 1, к.т.н., стариий научный сотрудник, master_v@inbox.ru \\ A.H. Фомин ${ }^{1}$, к.m.н., директор,mr.fominan@yandex.ru \\ 1 Научно-исследовательский технологический институт им. С.П. Капииь Ульяновского \\ государственного университета, г. Ульяновск, 432017, Россия
}

В статье описана структура программно-аппаратного комплекса для удаленной диагностики наземных транспортных средств с использованием канала связи GSM. Актуальность разработки данного устройства обусловлена потребностью в оперативном контроле и статистической обработке большого объема диагностической информации. Появление единого стандарта в начале 2000-х годов позволило охватить универсальными средствами диагностики основное количество транспортных средств, не углубляясь в конструктивные особенности каждого из них.

В работе показаны базовая схема взаимодействия элементов программно-аппаратного комплекса и структурная схема аппаратной части диагностического адаптера. Конструктивно диагностический адаптер состоит из нескольких модулей, управляемых с помощью двух микроконтроллеров семейства STM32 фирмы STMicroelectronics. Один из них выполняет функции контроллера автомобильных интерфейсов и обеспечивает физический обмен данными с электронными автомобилями. Функции второго контроллера заключаются в обработке и передаче данных на удаленный сервер. При этом осуществляется привязка получаемых диагностических данных к географическому положению транспортного средства, вычисляемого с помощью встроенного в адаптер модуля GPS, что позволяет выявить взаимосвязь между условиями эксплуатации и количеством возникающих неисправностей.

Представлена подробная схема программного взаимодействия аппаратных и программных компонентов комплекса, а также описаны принципы взаимодействия между диагностическим адаптером, установленным на транспортном средстве, и удаленным сервером, предназначенным для сбора, хранения и обработки диагностической информации. Программная реализация серверной составляющей комплекса включает в себя два модуля, объединенных общей БД. Задача одного из модулей - обеспечение пользовательского веб-интерфейса. Второй модуль реализует диагностические алгоритмы.

Ключевые слова: диагностика транспортных средств, ОВD-II, микроконтроллеры, электронный блок управления двигателем, STM32.

Современные транспортные средства оснащаются большим количеством электронных модулей, обеспечивающих функционирование основных агрегатов. Ключевое место среди таких модулей занимает электронный блок управления (ЭБУ) двигателем, в задачи которого входят функции генерации управляющих сигналов системы зажигания и подачи топлива путем контроля за показаниями различных датчиков. Полученная блоком управления информация позволяет обеспечить работу двигателя в рамках заданных мощностных и экологических показателей.

Техническая сложность электронных систем впрыска топлива, как правило, подразумевает их диагностику в условиях специализированных ремонтных предприятий, а чаще всего дилерских центров. В условиях недостаточно развитой сети дилерских центров такое решение имеет очевидные недостатки, заключающиеся в необходимости доставки транспортного средства, отсутствии оперативного контроля состояния автомобиля и существенных материальных затрат.

В начале 2000-х годов были приняты стандарты, обязывающие всех мировых автопроизводителей устанавливать электронные блоки, позволяющие контролировать основные параметры работы двигателя. Был стандартизирован диагностический протокол OBD-II (On-board diagnostics) [1], с помощью которого эти параметры можно считать с электронного блока, а также разработан универсальный разъем для подключения диагностического оборудования [2].

Современный уровень развития телекоммуникационных технологий позволяет эффективно передавать большие объемы информации в реальном времени, что открывает возможности для удаленной диагностики и контроля транспортных средств, в том числе в автоматическом режиме. В этом случае диагностика осуществляется удаленным сервером на основе накопленных и оперативных данных.

В рамках данной работы были поставлены следующие задачи:

- разработка алгоритма экспертного анализа диагностической информации, передаваемой с помощью телеметрии;

- разработка алгоритма интерактивного анализа диагностической информации и представления оценок состояния бортовых систем транспортных средств;

- формирование БД штатных и нештатных состояний бортовых систем транспортных средств для последующей обработки с помощью экспертного анализа; 
- выявление критериев идентификации аномальных показателей и отклонений от типовых эксплуатационных значений.

Для решения этих задач разработан программноаппаратный комплекс, состоящий из адаптера, подключаемого к диагностическому разъему транспортного средства, и удаленного сервера, обеспечивающего обработку диагностической информации. Базовая схема взаимодействия элементов программноаппаратного комплекса показана на рисунке 1.

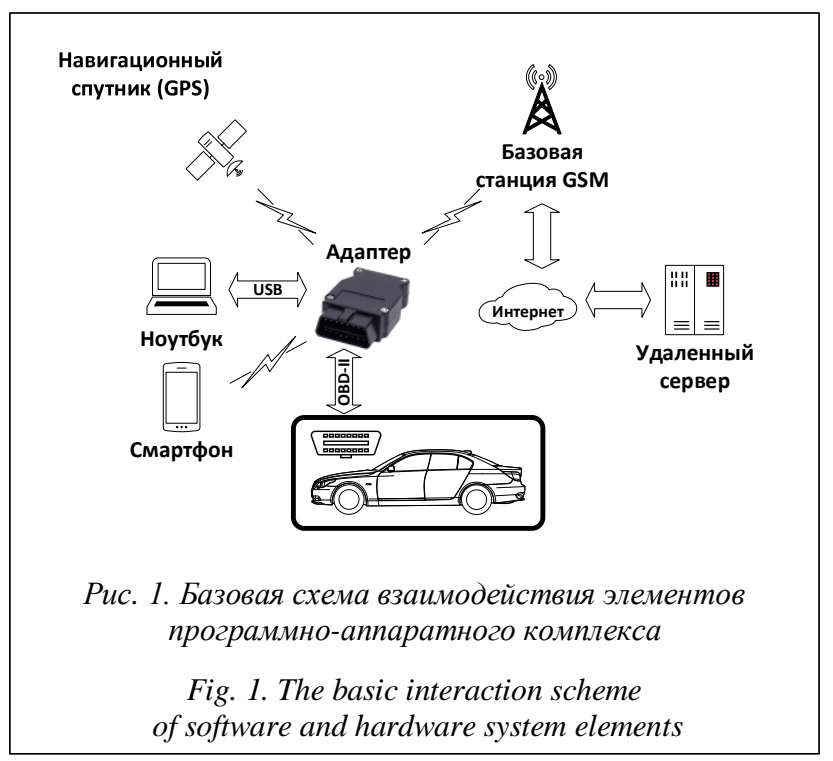

Адаптер получает питание от бортовой сети автомобиля и осуществляет сбор диагностических данных, которые могут быть обработаны локально (через беспроводное соединение Wi-Fi на смартфоне или ноутбуке) либо переданы на удаленный сервер по каналу GSM. Адаптер оснащен системой геопозиционирования для приема сигналов навигационных спутников GPS (рис. 2).

Диагностический адаптер программно-аппаратного комплекса оснащается двумя высокопроизводительными микроконтроллерами STM32F103C8T6 [3]: главным контроллером, выполняющим функции обработки получаемой диагностической информации и информации о местоположении, а также передачи ее по каналам связи, и контроллером автомобильных интерфейсов, обеспечивающим работу универсального диагностического устройства с различными типами автомобильных протоколов (ISO15765-4 (CAN), ISO14230-4 (KWP2000 или К-линия), ISO9141-2 (К-линия), J1850 VPW, J1850 РWM и др.).

Применение индивидуальных отладочных интерфейсов для каждого контроллера позволяет осуществлять эффективную отладку встраиваемого ПО. Структурная схема программного взаимодействия компонентов диагностического адаптера показана на рисунке 3.

Включение устройства сопровождается подачей питания ко всем основным его компонентам: к глав-

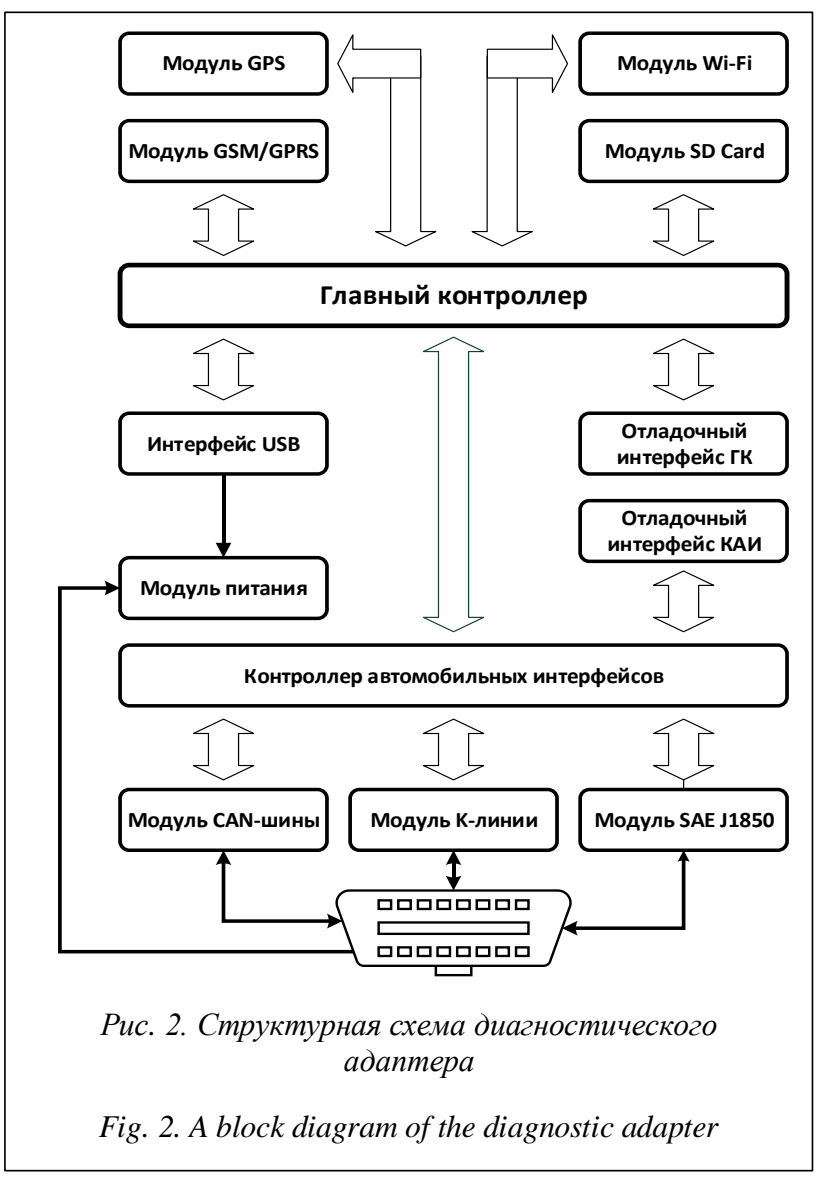

ному контроллеру, контроллеру автомобильных интерфейсов, к модулям GPS и Wi-Fi.

Процесс инициализации главного контроллера включает в себя загрузку встроенного ПО, которое производит настройку внутренних параметров (тактового генератора, портов ввода-вывода общего назначения, коммуникационных интерфейсов) и определяет алгоритм функционирования контроллера.

Инициализация контроллера автомобильных интерфейсов происходит аналогичным образом. Управляющая программа настраивает периферию контроллера и переходит в режим ожидания команд от главного контроллера по протоколу SPI. Инициализация модуля GPS подразумевает переход в состояние уверенного получения сигнала от навигационных спутников (режим hot) и начало передачи главному контроллеру геопозиционных данных по коммуникационному интерфейсу USART с помощью протокола NMEA [4]. Инициализация модуля беспроводной связи Wi-Fi определяется временем выхода блока в режим готовности к принятию АТ-команд по коммуникационному интерфейсу USART. После окончания инициализации главный контроллер осуществляет включение GSMмодуля, который начинает свою процедуру инициализации. Главный контроллер ожидает ответа GSMмодуля и состояния его готовности к работе с сетью сотового оператора, а затем переходит к основному циклу работы. Основной цикл работы включает в себя 


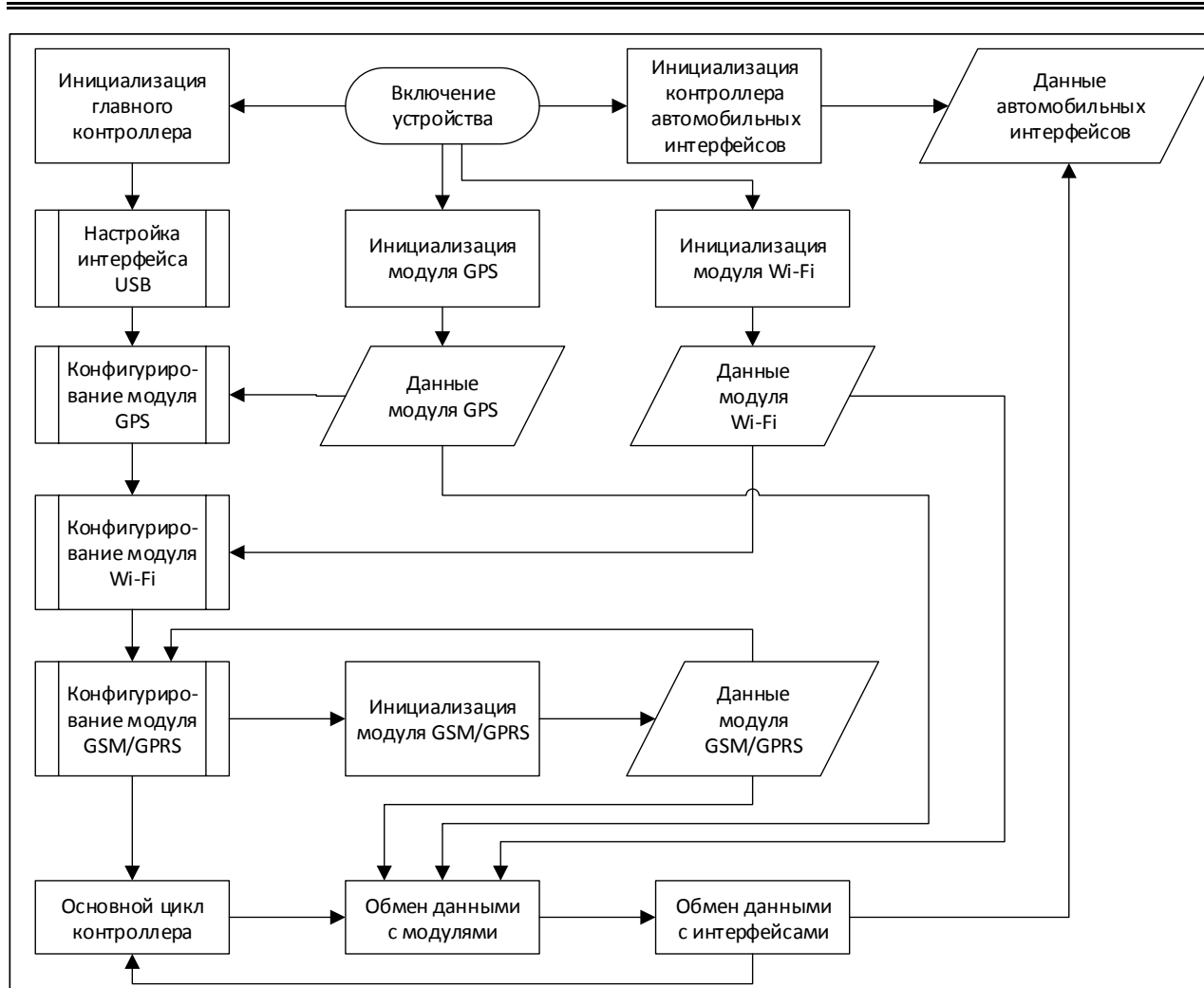

Рис. 3. Структурная схема программного взаимодействия компонентов диагностического адаптера

Fig. 3. A block diagram of software interaction of diagnostic adapter components

тификацию поступающей информации, осуществляют программное управление и регулирование работы диагностических адаптеров. Взаимодействие сервера обработки диагностической информации с диагностическими устройствами из внешней сети защищается с помощью брандмауэра. Сервер обработки диагностической информации включает в себя программный модуль, выполняющий функции краткосрочного прогнозирования [7] и интерполяции [8] значений ключевых параметров силового агрегата транспортного средства (топливная коррекция, температура и другие).

3. Сервер БД, представляющий собой СУБД, предназначенную для накопления полученной диагностиче-

обработку поступающей от модулей информации, в том числе управляющих команд, и взаимодействие с контроллером автомобильных интерфейсов с целью обмена диагностическими данными с электронными блоками автомобиля.

Концепция построения серверной составляющей комплекса показана на рисунке 4.

Функционально серверная сторона состоит из трех основных частей.

1. Веб-сервер, обеспечивающий работу пользовательского (клиентского) интерфейса, расположенного на стационарных компьютерах, ноутбуках, смартфонах и т.д. В его задачу входит взаимодействие с сервером БД для получения актуальной информации (диагностические, статистические и геопозиционные данные) для каждого клиента. Взаимодействие вебсервера с клиентами из внешней сети защищается с помощью брандмауэра.

2. Сервер обработки диагностической информации, предназначенный для взаимодействия с адаптерами, установленными на транспортных средствах. Сервер осуществляет передачу управляющих команд и прием диагностических и геопозиционных данных и выполняет передачу полученной информации на сервер БД. Алгоритмы сервера обработки диагностической информации базируются на методах системного анализа [5, 6]: выполняют распознавание и иден- ской и геопозиционной информации. Сервер БД является связующим звеном программных компонентов системы и может быть использован для получения статистических данных, на основе которых осуществляется прогноз остаточного ресурса и надежности транспортного средства [9].

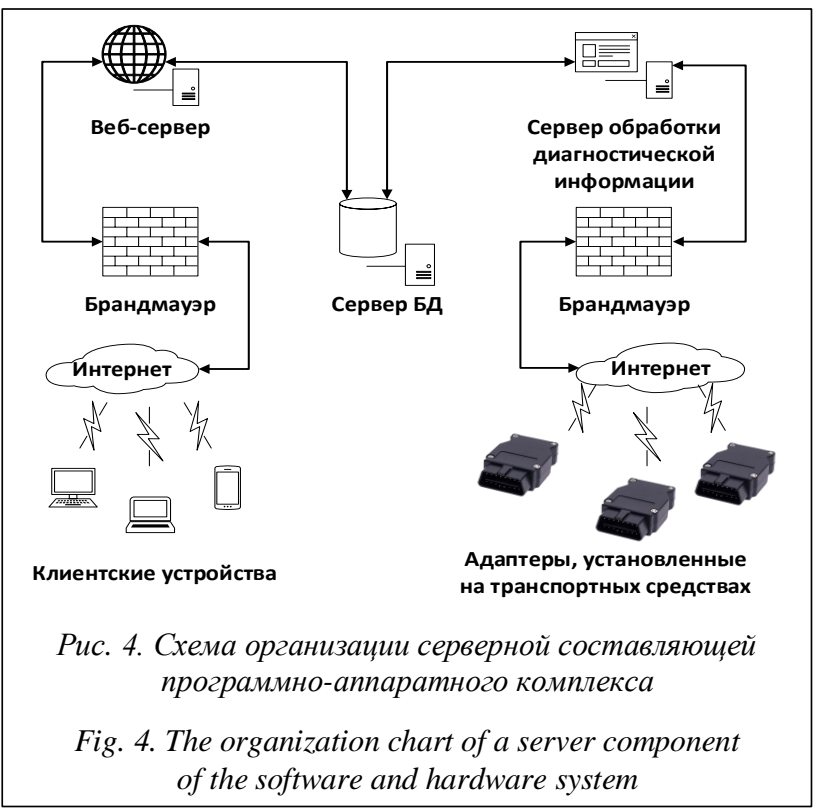




\section{Выводы}

Разработанный программно-аппаратный комплекс позволил решить ряд задач, нацеленных на улучшение технико-экономических показателей двигателя внутреннего сгорания. В частности, проведена верификация авторских алгоритмов экспертного анализа диагностической информации, отработан алгоритм интерактивного анализа диагностической информации и накоплена БД нештатных ситуаций для формирования критериев идентификации аномальных показателей.

\section{Лumepamypa}

1. Dzhelekarski P., Alexiev D. Initializing Communication to Vehicle OBDII System. Proc. Intern. Conf. ELECTRONICS, 2005, vol. 3, pp. 46-52.
2. Malekian R., Moloisane N.R., Nair L., Maharaj B.T., ChudeOkonkwo U.A.K. Design and Implementation of a Wireless OBD II Fleet Management System. IEEE Sensors J., 2017, vol. 17, no. 4, pp. 1154-1164.

3. Zhang H., Kang W. Design of the Data Acquisition System Based on STM32. Procedia Comp. Sc., 2013, vol. 17, pp. 222-228.

4. Si H., Aung Z.M. Position Data Acquisition from NMEA Protocol of Global Positioning System. IJCEE, 2011, vol. 3, no. 3, pp. 353-357.

5. Блауберг И.В. Проблема целостности и системный подход. М.: Едиториал УРСС, 1997. 450 c.

6. Робустов В.В. Системный анализ факторов влияния на успех пуска ДВС в условиях отрицательных температур // Омский науч. вестн. 2006. № 3. С. 100-104.

7. Лукашин Ю.П. Адаптивные методы краткосрочного прогнозирования временных рядов. М.: Финансы и статистика, 2003. $416 \mathrm{c}$.

8. Воеводин В.В., Воеводин Вл.В. Параллельные вычисления. СПб: БХВ-Петербург, 2002. 608 с.

9. Зудов Г.Ю., Ишков А.М., Левин А.И. Методика расчета остаточного ресурса автомобиля // Вестн. ИрГТУ. 2014. № 12. C. $161-164$.

\section{Developing a software and hardware system for GSM remote diagnostics of surface vehicles}

D.S. Lavygin ${ }^{1}$, Ph.D. (Engineering), Senior Researcher

V.V. Levshchanov ${ }^{1}$, Ph.D. (Engineering), Senior Researcher, master_v@inbox.ru

A.N. Fomin ${ }^{1}$, Ph.D. (Engineering), Director, mr.fominan@yandex.ru

${ }^{1}$ Technological Research Institute of Ulyanovsk State University, Ulyanovsk, 432017, Russian Federation

Abstract. The paper describes the structure of a software and hardware system for remote diagnostics of surface vehicles by a GSM channel. The developed device is relevant due to the need of operational control and statistical processing of a large amount of diagnostic information. A unified standard, which appeared in the early 2000 s, made it possible to diagnose a number of vehicles by universal tools without considering design features of each of them.

The paper shows the basic scheme of interaction between hardware and software elements of the system and the structural diagram of a diagnostic adapter hardware part. The diagnostic adapter device consists of several modules controlled by two STM32 microcontrollers (STMicroelectronics). One of them controls automobile interfaces and provides data exchange with electronic equipment of a vehicle. The second controller processes and transfers data to a remote server. At the same time, the obtained diagnostic data is linked to a vehicle geographic location (calculated using the built-in GPS module), which allows identifying the relation between operating conditions and a number of faults that occur.

The paper also shows a detailed scheme of software interaction between hardware and software components of the complex, and describes interaction principles between the vehicle diagnostic adapter and a remote server for collecting, storing and processing diagnostic information. The software implementation of the server component includes two modules united by a common database. The first module provides a user-friendly web interface. The second one implements diagnostic algorithms.

Keywords: vehicle diagnostics, OBD-II, microcontrollers, engine control module, STM32.

\section{References}

1. Dzhelekarski P., Alexiev D. Initializing communication to vehicle OBDII system. Proc. Intern. Conf. ELECTRONICS'2005. Sozopol, Bulgaria, 2005, vol. 3, pp. 46-52.

2. Malekian R., Moloisane N.R., Nair L., Maharaj B.T., Chude-Okonkwo U.A.K. Design and implementation of a wireless OBD II fleet management system. IEEE Sensors J. 2017, vol. 17, no. 4, pp. 1154-1164.

3. Zhang H., Kang W. Design of the Data Acquisition System Based on STM32. Procedia Comp. Sci. 2013, vol. 17, pp. 222-228.

4. Si H., Aung Z.M. Position data acquisition from nmea protocol of global positioning system. IJCEE. 2011, vol. 3, no. 3, pp. 353-357.

5. Blauberg I.V. The Integrity Problem and System Approach. Moscow, Editorial URSS Publ., 1997, 450 p.

6. Robustov V.V. System analysis of factors influencing the success of internal combustion engine start-up during negative temperature conditions. Omsk Sci. Bulletin. Omsk, 2006, no. 3, pp. 100-104 (in Russ.).

7. Lukashin Yu.P. Adaptive Methods for Short-Term Forecast of Time Series. Moscow, Finansy i statistika Publ., 2003,416 p.

8. Voevodin V.V., Voevodin Vl.V. Parallel Computing. St. Petersburg, BHV-Peterburg Publ., 2002, 608 p.

9. Zudov G.Yu., Ishkov A.M., Levin A.I. The methodology for calculating remaining vehicle lifetime. Proc. of Irkutsk State Technical Univ. 2014, no. 12, pp. 161-164 (in Russ.). 